NANO: Brief Reports and Reviews

Vol. 10, No. 7 (2015) 1530005 (11 pages)

(C) World Scientific Publishing Company

DOI: $10.1142 /$ S1793292015300054

\title{
Classical Plasmonics: Wave Propagation Control at Subwavelength Scale
}

\author{
Víctor Coello and Cesar E. Garcia-Ortiz \\ CICESE Monterrey, Alianza Centro No. 504 PIIT \\ Apodaca, N. L. C. P. 66629, Mexico \\ Manuel Garcia-Mendez \\ CICFIM FCFM \\ UANL. Ave Universidad $S / N$. Cd. Universitaria \\ San Nicolás de los Garza, N. L. México \\ Received 4 February 2015 \\ Accepted 27 May 2015 \\ Published 14 July 2015
}

\begin{abstract}
In this paper, surface plasmons polariton propagation and manipulation is reviewed in the context of experiments and modeling of optical images. We focus our attention in the interaction of surface plasmon polaritons with arrays of micro-scatereres and nanofabricated structures. Numerical simulations and experimental results of different plasmonic devices are presented. Plasmonic beam manipulation opens up numerous possibilities for application in biosensing, nanophotonics, and in general in the area of surface optics properties.
\end{abstract}

Keywords: Plasmonics; subwavelength phenomena; nanostructures.

\section{Introduction}

\subsection{General aspects of surface plasmon polaritons}

Classical Plasmonics is a very active research area dealing with fundamental studies of surface plasmon polaritons (SPPs), ${ }^{1,2}$ and opening promising technological perspectives within nano-optics, ${ }^{3}$ metamaterials, ${ }^{4}$ life science ${ }^{5}$ among other areas. SPPs are oscillations of surface electron charge density that exist at a metal/dielectric interface. Such oscillations are traveling waves with well-defined frequency, wave vector, and are evanescently confined in the perpendicular direction to the propagation. Due to their electromagnetic nature, SPPs can diffract, reflect, and interfere. These properties are clearly exhibited in the course of SPP propagation. Local control of SPP propagation has always been a difficult task. The reason being, at least partially, because is easier by far to scatter SPPs out of the surface plane, in the form of propagating waves, than along to it, since the maximum intensity of the SPP field is located at the surface. Attenuated total internal reflection (ATR) spectra ${ }^{6}$ have been typically used in order to evaluate the SPP propagation at a metallic/dielectric interface. In spite of being versatile and easy to implement the technique, the SPP field measurements are carried out in an indirect form which limits its scope. A direct probe of SPP fields was only possible when an idea proposed by Synge ${ }^{7}$ in 1928 about overcoming the diffraction limit of resolution was brought into an 
experiment $^{8}$ : The so-called scanning near field optical microscopy (SNOM).

\subsection{Near-field imaging of surface plasmon polaritons}

The birth of the SNOM technique, which has its basis in the detection of evanescent fields, had a big impact on the SPP research activity. Some of the pioneering investigations, in this context, were related to SPP propagation along thin metal films with inherent surface imperfections. ${ }^{9}$ SPP propagation (and its subsequent scattering) leads to generally complex near-field intensity variations across a surface ${ }^{9}$ which may even become misleading as the volume fraction of surface defects becomes larger. ${ }^{10}$ Nevertheless, a relatively large density of surface scatterers is necessary in order to affect the transport of light and therefore produce phenomena such as SPP localization ${ }^{10}$ which remains nowadays as one of the most exciting studies related to this research field. A correct interpretation of those near-field signals, once they are recorded, is a significant problem especially when the SNOM probe is acting as a nonpassive element ${ }^{11}$ of the optical fields. In order to deal with it, exhaustive investigations of probe-surface distances dependence of near field optical signals, with the SPP being resonantly excited, were performed. ${ }^{12}$ Such procedure allows to evaluate the influence of propagating components on the SPP near field distributions, and to determine whether it is sufficiently small to neglect it. Therefore, by routinely verifying that the detected signal is primarily related to the total SPP field intensity, one can claim that the near-field images are directly related to the phenomenon under study.

\subsection{Micro and nano-optics of surface plasmon polaritons}

Such progress in understanding local measurements of SPP properties stimulated research in various directions, one of them being investigations of local control of SPP propagation. The similitude between SPPs and waves propagating in planar waveguides (both are two-dimensional (2D) waves propagating in the surface) evokes the fascinating idea of an integrated optics of SPPs. Such an idea started to take form when reports about controlling SPP propagation, by using artificially created individual surface defects while continuously adjusting their shapes and sizes, were presented by the first time. ${ }^{12-15}$ These initial investigations confirmed the possibility to create components such as line and corner square SPP mirrors, and eventually combine them with spatially resolved SPP excitation. A remarkable progress in this field was achieved with the extension of the photonic band gap (PBG) effect to the plasmonic area. ${ }^{16}$ The concept of PBG is deeply rooted on that of Bragg diffraction and predicts a high control of the $2 \mathrm{D}$ propagation of SPPs ${ }^{17}$ as well as the possibility of achieving almost a perfect mirror effect. ${ }^{18}$ Furthermore, an idea based on the similarities between the PBG effect and the phenomenon of strong SPP localization was proposed as an interesting alternative mechanism of SPP guiding. ${ }^{19}$ On the other hand, the combination of numerical simulations with experimental methods is one focus of the plasmonic research activities. ${ }^{20}$ Numerical simulations performed with a relative simple vectorial dipolar model for multiple SPP scattering were used as a complementary research in order to realize a $2 \mathrm{D}$ counterpart of conventional optics. ${ }^{21}$ For example, from the calculations of a plasmonic interferometer element, it was understood that the splitting efficiency is very sensitive to the incidence angle, ${ }^{22}$ which is in good agreement with experimental data available in literature. ${ }^{23}$ Another interesting alternative for investigation of plasmonic elements is the usage of microwave radiation. Such electromagnetic range makes "scaling" of the optical problem under question possible. Thus, it was proposed a simple SNOM in the microwave range that was designed to adopt dissimilar illumination operations modes and with capabilities for studies of potential 2D optical devices. ${ }^{24,25}$

\subsection{Recent advances and perspectives in plasmonics}

More recently, a renewed impulse to the fundamental and technological research of the plasmonic area came with the development of the leakage radiation microscopy (LRM). ${ }^{26}$ The technique is simple in conception and provides a rapid $2 \mathrm{D}$ SPP mapping, which is an important difference in functionality between SNOM and LRM techniques. Furthermore, LRM presents the possibility of simultaneous access to direct and reciprocal space. LRM has stimulated plasmonics research in various directions, one of them being, precisely, SPP 
interaction and manipulation with nanostructures. ${ }^{27-32}$ In LRM, conventionally, the SPP excitation is achieved by normal light illumination on a ridge that is located at the top of an air/metal interface. ${ }^{33}$ The excitation technique has some disadvantages for photonic integrated circuits since the incoupling ridge represents an additional interfacing element. In an attempt to avoid such extra element, the possibility of a simultaneous process of SPP excitation and propagation control was numerically elucidated. ${ }^{34}$ The proposed system could act as, both, the launching mechanism and the plasmonic element itself. A similar mechanism is usually adopted in plasmonic waveguides, where in the buswaveguide is included a taper, at one of its ends, that is used to couple light efficiently into SPP waveguide mode by directly illuminating the taper region. ${ }^{35,36}$ Despite the apparent success in the broad variety of plasmonic waveguide elements so far reported, ${ }^{37}$ all the systems show a trade-off between subwavelength confinement and propagation loss. In other words, increasing the confinement factor automatically results in a diminution of the mode propagation length. In order to deal with this, gain media have been proposed to compensate ohmic loss through optical amplification. ${ }^{38-42}$ However, it is in general rather difficult to obtain loss compensation and the search for new, more promising, active media for amplification of plasmonic modes remains to be an open problem. Another interesting approach to overcome this tradeoff between confinement and propagation could be the generation of diffraction-free plasmonic beams ${ }^{43-49}$ (DF-SPPBs). However, the very existence of DFSPPBs solutions to the free space three-dimensional (3D) Helmholtz equation is not straight forwardly warranted. In fact, it was shown that the Airy beam is the only possible diffraction-free solution to the 2D paraxial wave equation. ${ }^{50}$ Nevertheless, numerical studies in photonic crystals demonstrated that diffraction can be tailored to generate $2 \mathrm{D}$ diffraction free beams that resemble the profile of a Bessel function and present the self-healing property. ${ }^{51}$

Here, we present an overview of some of the problems, developments, and current progress related with our research in the plasmonics wave propagation control. We begin with an introduction to far-field characterization of SPPs properties in Sec. 2 followed by a brief discussion of SPP microoptic concepts in Sec. 3. Numerical results on light to SPP coupling will be presented in Sec. 4 .
Section 5 describes a special kind of plasmonic structures that were used for SPP waveguiding. Direct evidence of diffraction-free plasmonic beams is presented in Sec. 6. Finally, in Sec. 7, applications and further investigations of SPP propagation and manipulation are outlined.

\section{Far-Field Characterization of SPP Properties}

SPP light excitation, at an ideal flat interface, is not possible to reach without a coupler since SPP wavenumber is larger than that of the light illumination. Coupling of photons into SPPs can be achieved either by using a grating coupler, ${ }^{6}$ or by means of ATR devices such as: Otto and Kretschmann configurations. ${ }^{6}$ The classical ATR techniques are still used in a broad number of SPP studies including plasmonic metamaterials ${ }^{4}$ and SPP resonance based sensors. ${ }^{5}$ They consist of a dielectricmetal-air interface where a light beam is impinging, on the metallic surface, under an angle larger than the critical angle for that interface. The SPP excitation is recognized as a minimum in the angular dependence of the reflected beam power (ATR curve). The ATR curve (Fig. 1) allows one to deduce SPP characteristics such as propagation length $(L)$, penetration depths in air and film $\left(d_{\text {air }}\right.$ and $\left.d_{\text {metal }}\right)$, SPP wavelength, $\lambda_{\mathrm{SPP}}$, as well as the dielectric constant, $\varepsilon$, of the metal film. A precise knowledge of those characteristics is indispensable for any kind of plasmonic studies. The ATR spectra of Fig. 1 were measured by using an illumination

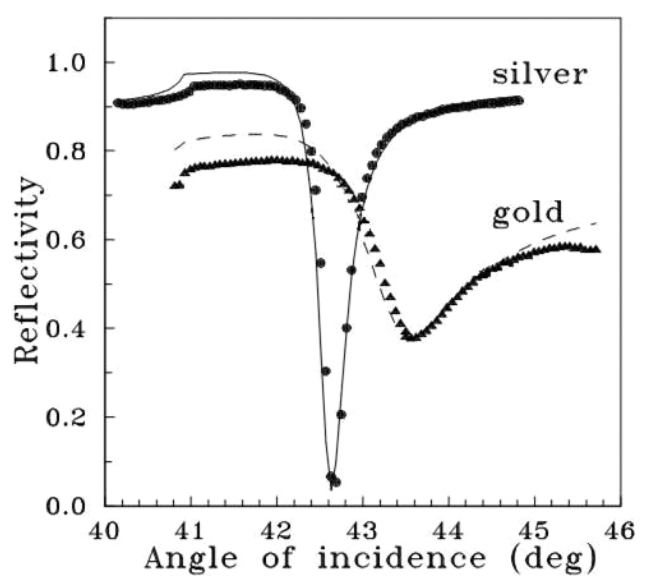

Fig. 1. Angular excitation spectra of surface plasmon polaritons measured (filled symbols). Illumination wavelength $\lambda=632.8 \mathrm{~nm}$. 
Table 1. Dielectric constants of metal films and various SPP characteristics at two thin films: calculated (solid lines) for silver $(45 \mathrm{~nm})$ and gold $(53 \mathrm{~nm})$ thin films. Illumination wavelength $\lambda=632.8 \mathrm{~nm}$.

\begin{tabular}{lccccc}
\hline $\begin{array}{l}\text { Sample } \\
\text { (Thickness) }\end{array}$ & $\varepsilon$ & $L$ & $d_{\text {air }}$ & $d_{\text {metal }}$ & $\lambda_{\text {SPP }}$ \\
\hline Silver $(45 \mathrm{~nm})$ & $-16+0.6 \mathrm{i}$ & $38 \mu \mathrm{m}$ & $390 \mathrm{~nm}$ & $25 \mathrm{~nm}$ & $612 \mathrm{~nm}$ \\
Gold $(53 \mathrm{~nm})$ & $-10.7+2.5 \mathrm{i}$ & $4.2 \mu \mathrm{m}$ & $320 \mathrm{~nm}$ & $30 \mathrm{~nm}$ & $602 \mathrm{~nm}$ \\
\hline
\end{tabular}

wavelength $\lambda=632.8 \mathrm{~nm}$ and for two metallic smooth films in order to determine the resonant excitation angle for the corresponding SPP. ${ }^{52}$ The shape of the ATR curve was quantitatively described by using the three layer Fresnel equation. The calculated curve allows one to extract the dielectric constant of the film from a fit to the measured resonance spectrum. In this fit, the film thickness, illumination wavelength, and prism refractive index were considered to be known. In order to fit the Fresnel formula to the reflectance data, we used a standard curve fit routine of a commercial software (Matlab). Thereby, we found various SPP characteristics which are showed in Table 1.

Such SPP characteristics are consistent with relatively recent reported values that were measured under similar conditions. ${ }^{53}$

Silver and gold films showed a very well pronounced minimum in their corresponding ATR spectra. Because the SPP resonance is strongly dependent on the refractive index of the sample, the spectra also showed an angular position displacement, with respect to each other, of the ATR minimum. In both cases, the SPP propagation distance is limited due to, both, ohmic losses in metal, which are accounted for in the imaginary part of $\varepsilon$, and scattering processes during course propagation. We should distinguish between two kinds of SPP scattering: inelastic and elastic SPP scattering (Fig. 2).

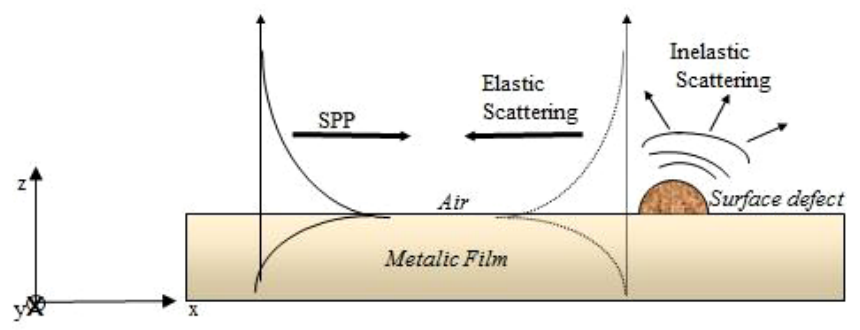

Fig. 2. Schematic representation of elastic and inelastic SPP scatering existing at a metal/dielectric interface.
Elastic scattering occurs when the SPP is scattered by surface imperfections along the surface plane, i.e., into another SPP preserving the total SPP energy (Fig. 2). Inelastic scattering, on the other hand, appears when the SPP is scattered into field components propagating away from the surface (Fig. 2). Inelastic scattering results in a decrease in the total energy that is stored in the SPP and therefore it is considered an unwanted effect. In addition, when imaging SPPs with SNOM techniques, the interference between the SPP fields and propagating waves may lead to a modification of the recorded optical image. In order to deal with the aforementioned contributions, we suggested to study in detail the probe-surface distance dependence of the optical image. In this context, let us consider a general representation of the total electric field $E_{\text {TOTAL }}$ (in air), that is generated due to the SPP propagating along a metal surface with the dielectric constant $\varepsilon=\varepsilon_{1}+\varepsilon_{2}$, in the absence of the probe, viz.,

$$
E_{\mathrm{TOTAL}}=E_{\mathrm{SP}}(x, y) e^{-\gamma \cdot z}+E_{\mathrm{PW}}(x, y, z),
$$

where $E_{\mathrm{SP}}(\mathrm{x}, \mathrm{y})$ represents the sum of the incident SPP and the SPPs elastically scattered by surface imperfections, $\gamma=\frac{2 \pi}{\lambda_{0}} \sqrt{\frac{1}{\varepsilon_{1}+1}}$ (assuming $\left.\left|\varepsilon_{1}\right| \gg\left|\varepsilon_{2}\right|\right)$, and $E_{\mathrm{PW}}(x, y, z)$ are propagating waves stemming from inelastic SPP scattering.

In the approximation of a passive probe ${ }^{54}$ the optical signal $P$ recorded with the SNOM is proportional to the square modules of the total electric field, and given by:

$$
P \propto\left|E_{\mathrm{SP}}\right|^{2} e^{-2 \gamma \cdot z}+2 \operatorname{Re} E_{\mathrm{SP}} E_{\mathrm{PW}}^{*} e^{-\gamma \cdot z}+\left|E_{\mathrm{PW}}\right|^{2} .
$$

It can be seen from Eq. (2) that the optical signal has, in general, three intensity distributions that vary differently with the distance to the surface. These contributions are drawn in Fig. 3 by using the experimental data for the analysis of the silver film that was under investigation (Table 1).

We obtained similar results, not shown here for brevity, for the case of the gold film (Table 1). The probe-surface distance dependence of Fig. 3 exhibits an exponential behavior for small probesurface distances. This means that once the SPP is being resonantly excited, the near field zone was dominated by evanescent fields of the excited and the elastically scattered SPPs, i.e., $E_{\mathrm{PW}} \ll E_{\mathrm{SP}}$ and 


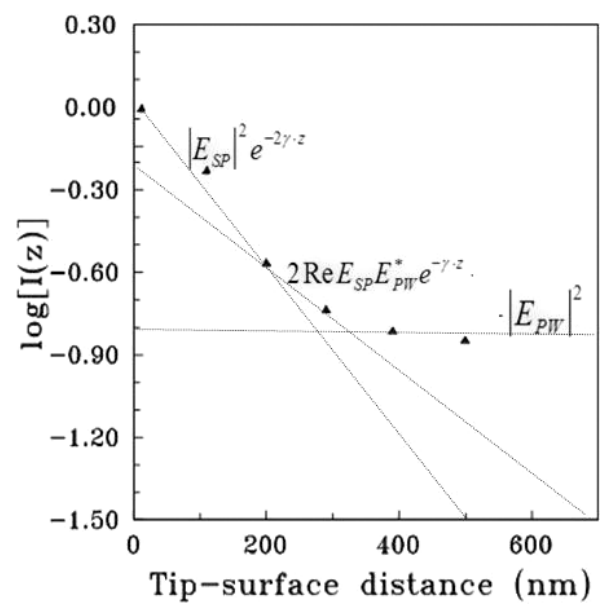

Fig. 3. Schematic and measured (filled symbols) tip-surface distance dependence of the optical signal for relatively weak propagating. The three different sections correspond to the different terms in Eq. (2).

therefore, one can claim that the near-field signal is directly related to the SPP intensity distributions.

\section{Micro-Optics of Surface Plasmon Polaritons}

One of the first techniques relied upon the fabrication of plasmonic structures made use of an uncoated fiber tip which was pressed against a metallic surface to create an elastic micro-scatterer. ${ }^{12}$ The whole setup consisted of a SNOM in photon tunnelling mode ${ }^{11}$ combined with a shear force feedback system ${ }^{55}$ and an arrangement for SPP excitation in the Kretschmann configuration. ${ }^{6}$ By lining up several bumps, with small separations between them, a common plane wavefront of the scattered light can be achieved. The idea was carried out for a 2-scatterer line array, whose inclination with respect to the applied field was $90^{\circ}$. The image for the line mirror showed the interference between a specular reflected SPP and the incident one, exhibiting a satisfactory behavior of the line mirror for normal incidence [Figs. 4(a) and 4(b)].

Nevertheless, line arrays were far from being perfect mirrors since much of the incident light passed through the structures [Fig. 4(b)]. This issue can be compensated by placing an array of lines that satisfy the Bragg condition, $2 d \sin \theta=n \lambda$, where $d$ is the separation distance, $\theta$ is the angle the beam makes with the mirror and $n$ is a whole number. The idea has been modeled using total Green's tensor formalism and the dipole approximation for

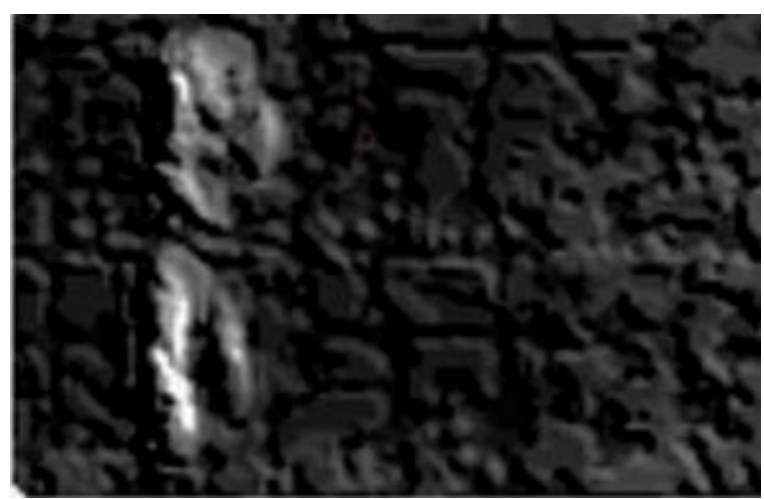

(a)

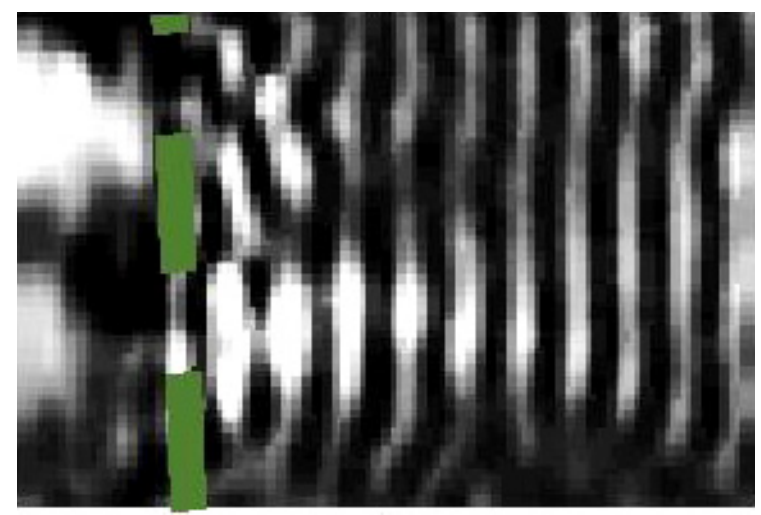

(b)

Fig. 4. Topographical (a) and near-field optical (b) images $\left(7.8 \times 7 \mu \mathrm{m}^{2}\right)$ obtained with a liner micromirrors (fabricated on the silver film). The dashed line is used to guide the eye.

multiple SPP scattering by nanoparticles. ${ }^{56,57}$ The bumps forming the arrays were approximated by spherical particles, considered as point-dipole scatterers suspended $40 \mathrm{~nm}$ above the surface, and characterized with isotropic free-space polarizability obtained in the long-wavelength approximation. Here, we chose an inter-particle distance of $200 \mathrm{~nm}$ and an inter-line separation of $350 \mathrm{~nm}$ with an incident beam angle of $60^{\circ}$. Then, the dipole moments of particles illuminated with a Gaussian SPP beam were calculated self-consistently, and then the total electric field distribution was determined above the surface (Fig. 5). Several other plasmonic systems that show a great potential for the development of nanophotonics circuits have been successfully implemented. An example of them is a plasmonic beam splitter. ${ }^{22}$ A beam splitter is an optical device that splits a beam of light in two. It is the crucial part of most interferometers. The plasmonic element was simulated by using again Green's tensor 


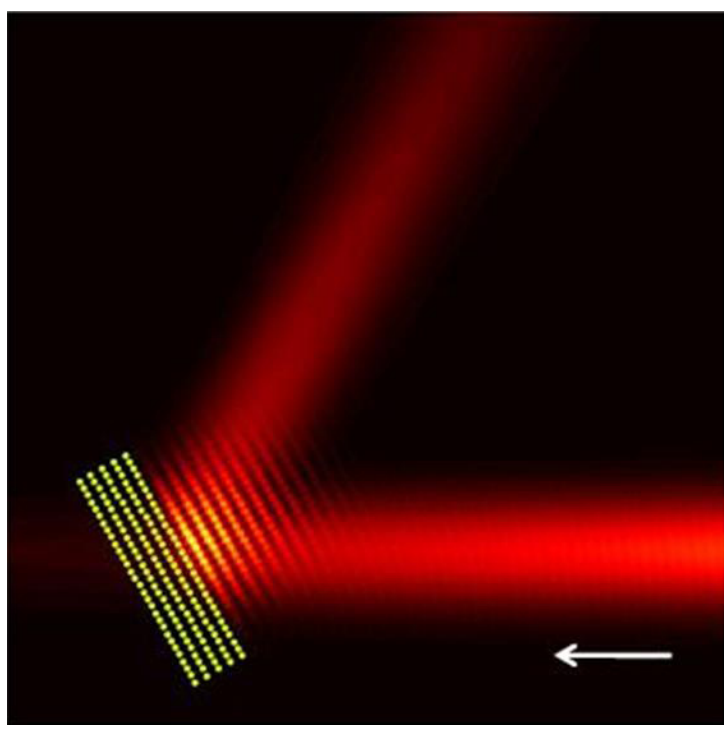

Fig. 5. Intensity distribution of a multiline mirror with five lines of 30 nanoparticles with radius $r=63 \mathrm{~nm}$. Inter-particle and inter-layer distances are $200 \mathrm{~nm}$ and $350 \mathrm{~nm}$, respectively. The incoming SPP beam has a wavelength, $\lambda_{0}=750 \mathrm{~nm}$. The circles symbols are used to guide the eye. The white arrow indicates the incident SPP propagation.

formalism and the dipole approximation for multiple SPP scattering by nanoparticles. In order to numerically built such a plasmonic bleamsplitter first, it was investigated the in-plane scattered field created by a 5 - $\mu$ m-wide Gaussian SPP beam $(\lambda=750 \mathrm{~nm})$ of a unit amplitude impinging on an equally spaced line of nanoparticles. The density of particles, the inter-particle distance as well as the particle size are all critical parameters for realizing the most suitable configuration for a nearly 50/50 SPP beam splitter. For example, the inter-particle distances were investigated in the range of 200$400 \mathrm{~nm}$. Additionally, several angles $\Theta_{0}$ of SPP beam incidence, with respect to the normal to the nanoparticles line were considered. Thus, it turns out that, for a line of 200 scatterers with interparticle distance of $280 \mathrm{~nm}$ and particle radius of $64 \mathrm{~nm}$, a nearly $50 / 50$ beam splitter is conceivable [Figs. 6(a) and 6(b)]. The angle of incidence was set to $\Theta_{0}=16^{0}$. Note that these values match well to the experimental reported ones ${ }^{23}$ including equivalence in volume of the experimental cylindrical scatterer and the calculated spherical particle in our case. In our simulation, the incident SPP propagates from left to right and the dielectric constant used was $\varepsilon=-23+1.4 \mathrm{i}$ which corresponds to gold at an illumination wavelength of $750 \mathrm{~nm}$.

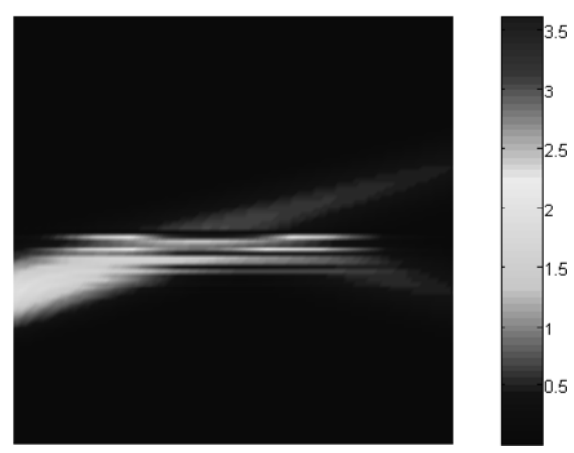

(a)

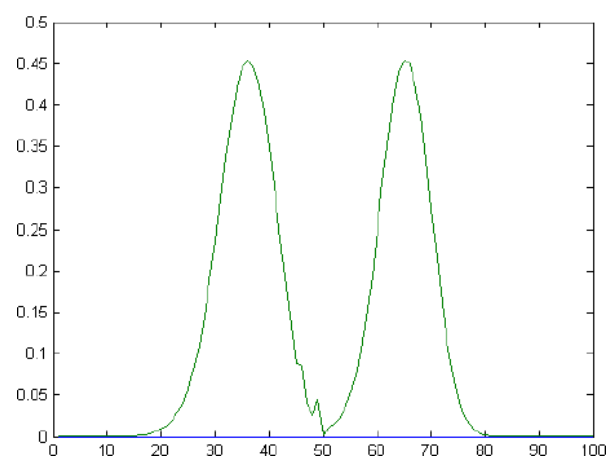

(b)

Fig. 6. SPP intensity distribution in the area of $50 \cdot 50 \mu \mathrm{m}^{2}$ calculated for a 50/50 SPP beamsplitter composed of 200 spherical particles with the radius of $64 \mathrm{~nm}$ separated by $280 \mathrm{~nm}$ and for the light wavelength of $750 \mathrm{~nm}$. The angle of incidence is set at $16^{\circ}$ with regard to the line of particles. The white arrow in (a) indicates the cross-section shown in (b).

\section{Light to SPP Coupling}

Based on the numerical modeling, we investigated the feasibility of exciting and manipulating SPPs without using external excitation elements. As we already mentioned, conventionally, most of SPP excitations methods are based on the use of glass prisms as couplers. ${ }^{6}$ An alternative is the use of a normal incident light to excite the SPPs through a ridge ${ }^{43}$ or plasmonic launching mechanism located at the top of an air/metal interface. ${ }^{43}$ Those approaches have, however, some drawbacks such as the large size of the prism couplers or the separation of the excitation source and the plasmonic devices which makes them not suitable for photonic integrated circuits. The simultaneous process of SPP excitation and propagation control was elucidated by using periodic square arrays of nanoparticle illuminated by a normally incident Gaussian beam. ${ }^{34}$ Therefore, the system is expected to work as, both, the launching mechanism and the 


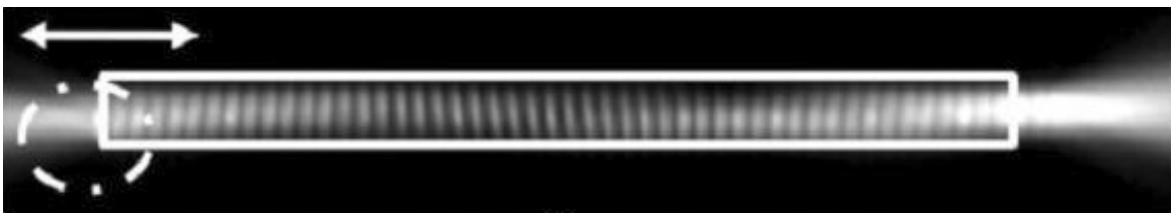

(a)

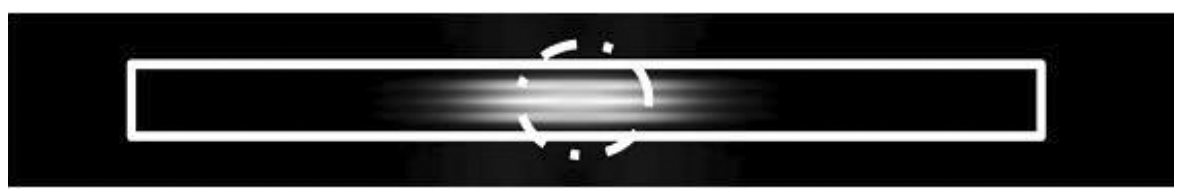

(b)

Fig. 7. Electric field magnitude distributions $\left(15 \times 2 \mu \mathrm{m}^{2}\right)$ calculated for nanoarray waveguides with $L=12 \mu \mathrm{m}, w=2 \mu \mathrm{m}$, $\Lambda=200 \mathrm{~nm}, r=20 \mathrm{~nm}$, and for incident beam positions placed at (a) low corner of left entrance (b) midsection, The dotted circle represents the incident Gaussian beam $(\mathrm{FWHM}=2 \mu \mathrm{m})$. The arrow indicates the incident light polarization in both cases.

plasmonic element itself. A nanodevice, realizable by a certain array structure, is a plasmonic waveguide. ${ }^{37}$ A particularly simple plasmonic waveguide is shown in Figs. 7(a) and 7(b). The waveguide consists of a periodic square-shaped nanoarray of $2 \mu \mathrm{m}$ width, $12 \mu \mathrm{m}$ length, inter-particle distance of $200 \mathrm{~nm}$ and particle radius of $20 \mathrm{~nm}$. We launched SPPs by illuminating, with a normally incident Gaussian beam $\left(\lambda_{0}=750 \mathrm{~nm}, \mathrm{FWHM}=2 \mu \mathrm{m}, \mathrm{x}-\mathrm{pol}\right)$, the low corner of the left-end of the nanoarray [Fig. 7(a)]. The SPP waveguiding capability is evidenced by the SPP beam coming out of the waveguide [Fig. 7(a)]. Note that, in Fig. 7(a), one can observe how the SPP is confined in the waveguide by multiple total internal reflections. By doing so, the SPP mode can be transported from one waveguide end to another one. In contrast, SPP waveguiding is almost not observed when the incident beam is placed on the midsection of the nanoarray [Fig. 7(b)]. At the midsection, the nanoarray is almost symmetric over the extent of the incident beam and therefore cannot scatter efficiently in the axial direction since the incoming propagating vector and the propagating SPP vector are hardly matched. However, this symmetry is broken at the nanoarrays ends where light is scattered into propagating SPP modes. Likewise, propagating SPP modes are excited in thin-film surface utilizing gratings or dots.

\section{Dielectric-Loaded Plasmonic Waveguides}

A major problem of plasmonic waveguides is associated with the weak confinement of the field in the transverse section. However, there are still opportunities for research in this context. For example, 'dielectric loaded SPP waveguides (DLSPPWs) ${ }^{37}$ have shown to be an attractive alternative for SPP waveguiding since they ensures a good tradeoff between mode confinement and propagation distance. Several waveguide devices based on DLSPPWs have been demonstrated so far. ${ }^{37}$ Among them, one can find interferometers, splitters, and waveguide ring resonators (WRRs). A WRR is a key building block in electronic-photonic integrated circuits. Furthermore, one can use waveguide-racetrack resonators (WRTRs) in order to increase the coupling efficiency of the WRRs. The larger coupling region of WRTRs yields flexibility in fabrication parameters, hence relaxing the requirements of fabrication resolution. By using electronbeam lithography, we have fabricated dielectric loaded plasmonic WRTRs operating at near-infrared wavelengths. ${ }^{35}$ One has to notice that at nearinfrared wavelengths, plasmonics properties such as propagation length, mode confinement and effective index dispersion are expected to be significantly different as compared with the ones of visible region. The structures consisted of a strip of poly-methyl-methacrylate (PMMA) deposited, by using spin-coating process, on a $70 \mathrm{~nm}$ gold film [Figs. 8(a) and 8(b)]. The width, w, and thicknesses, $t$, were both of $300 \mathrm{~nm}$ which ensure mono-modal conditions. ${ }^{58}$ The WRTRs consisted of a straight waveguide (bus-waveguide) in close proximity to a racetrack ring resonator which has two straight sections of length $L=1.4 \mu \mathrm{m}$ [Fig. 8(b)]. The transmission spectrum of the system was experimentally 


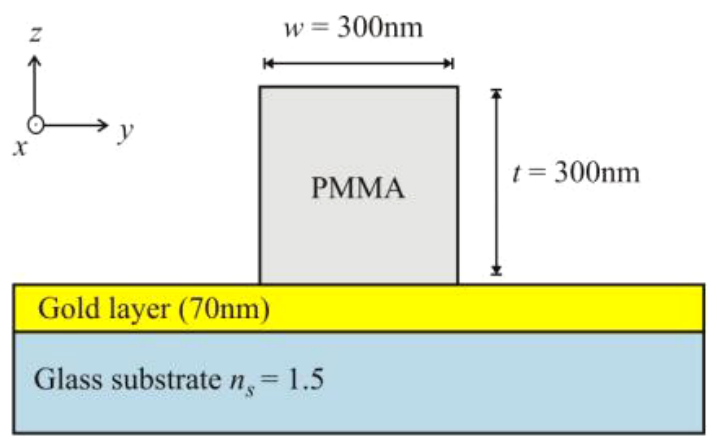

(a)

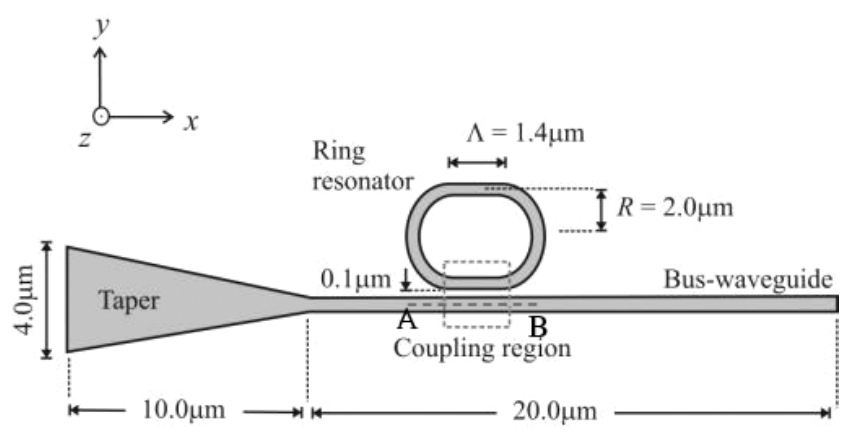

(b)

Fig. 8. Schematic diagram of the transverse section of a DLSPPW. (b) SPP waveguide racetrack resonator. The width $w$ and thickness $t$ of the WRTR is the same as shown in (a).

found by using LRM and compared to the calculated analytical expression. The transmission analytical expression ${ }^{59}$ of WRRs is given by:

$$
T=\exp \left(\frac{-l}{L_{S P}(\lambda)}\right) \frac{\alpha^{2}+t^{2}-2 \alpha t \cos \theta}{1+\alpha^{2} t^{2}-2 \alpha t \cos \theta} .
$$

The first factor of Eq. (3) corresponds to the exponential decay of the SPP with $l$ being the distance from A to B in Fig. 8. In the second factor of Eq. (3), $\alpha=\exp \left(-C \beta^{\prime \prime}\right)$ is a parameter accounting for the field attenuation per round trip around the resonator, where $\sigma$ is the pure bend loss factor and $C=$ $2 \pi R+2 \Delta$ is the circumference of the racetrack resonator, $t$ is the field transmission, which represents the coupling losses in the interaction region, and $\theta=(2 \pi / \lambda) n_{\text {eff }}(\lambda) C$ with $\lambda$ being the free-space wavelength. The direct-space leakage radiation images of the propagating mode inside the structures as well as the WRTR transmission spectrum are shown in Figs. 9(a)-9(e). The transmission values of a WRTR were experimentally measured by analyzing the input and output intensity located at the points A and B, respectively [Fig. 8(b)]. The
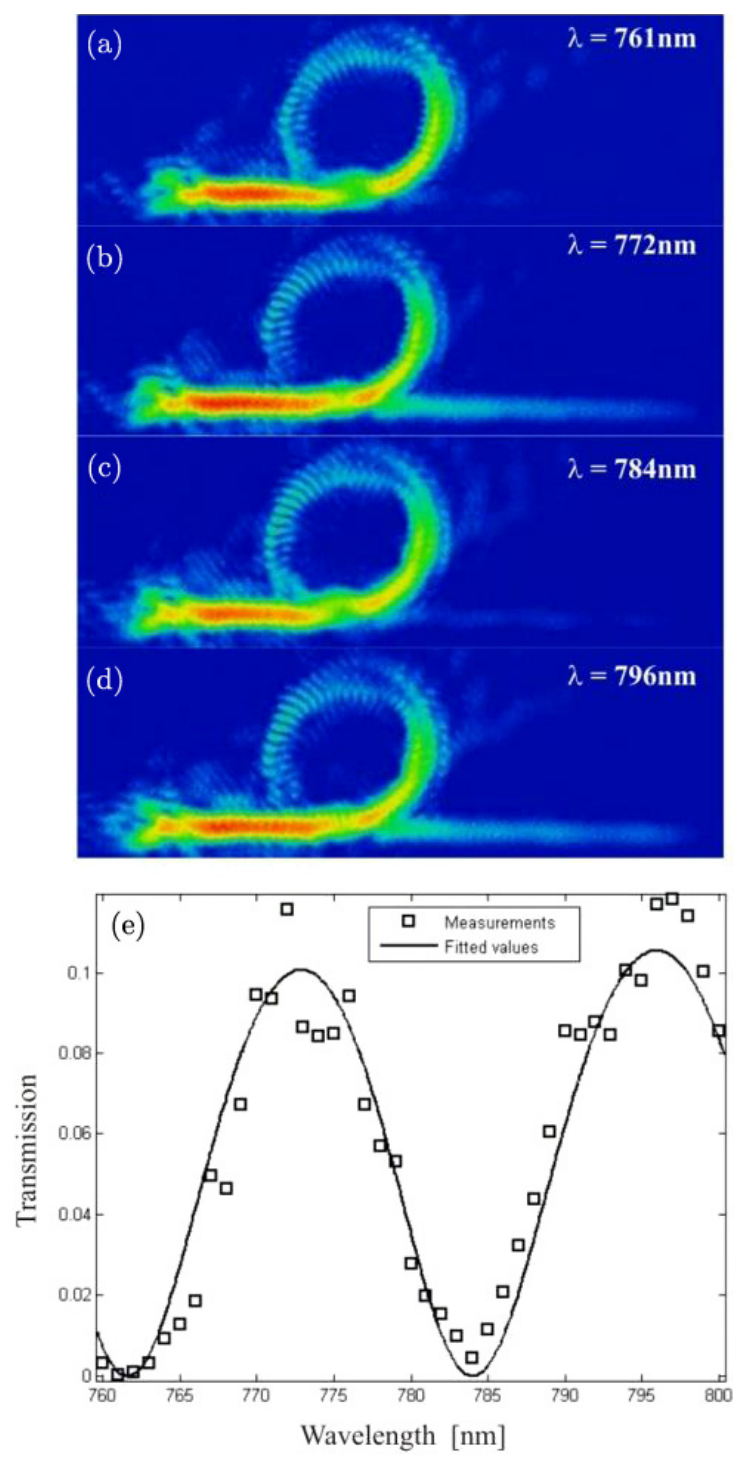

Fig. 9. WRTR LRM images illuminated with the free-space wavelength of (a) $761 \mathrm{~nm}$, (b) $772 \mathrm{~nm}$, (c) $784 \mathrm{~nm}$ and (d) $796 \mathrm{~nm}$. (e) WRTR transmission spectrum. Experimental measurements were obtained from LRM image analysis and the fitted values are found from the analytical expression.

values are found by obtaining the averaged intensity profiles at these points. Transmission minima occur close to $\lambda=761 \mathrm{~nm}$ and $784 \mathrm{~nm}$ [Figs. 9(a) and 9(c)] and maxima were around $\lambda=772 \mathrm{~nm}$ and $796 \mathrm{~nm}$ [Figs. 9(b) and 9(d)]. The experimental measurements of transmission were plotted as a function of wavelength [Fig. 9(e)]. Measured data exhibit an extinction ratio of $\sim 20 \mathrm{~dB}$ and a bandwidth of $\sim 11 \mathrm{~nm}$. The measured and calculated values of the WRR transmission [Fig. 9(e)] were numerically processed to find the best fit. The wavelengthdependent parameters of the analytical expression, 
i.e., the propagation length and effective index dispersion, were experimentally estimated by leakage radiation imaging of direct and Fourier space, respectively. The fitted values were $C=$ $15.09 \pm 0.01 \mu \mathrm{m}, \sigma=1.0 \pm 0.2$ and $t=0.32 \pm 0.04$ (the uncertainty intervals account for $95 \%$ of confidence). $\alpha$ was also calculated from 0.22 to 0.25 , due to its wavelength dependence. The fitted value of the ring perimeter $C$ differs only by $\sim 300 \mathrm{~nm} \quad(<2 \%)$ from the original fabrication parameters and the fitted value of $\sigma$, very close to unity, corresponds to very low bend losses.

\section{Difraction-Free Plasmonic Beams}

As we have seen, one of the most attractive properties of SPPs is that they can be concentrated and guided in nanostructures. However, in general, SPP waveguiding is still a challenge. Any diffraction directly results in loss as the spreading of the wave reduces the coupling between the components. A good alternative to such issue could be the generation of diffraction-free plasmonic beams (DFSPPBs). In general, diffraction-free beams are special kind of beams whose central maxima are remarkably resistant to the diffractive spreading commonly associated with all wave propagation. Furthermore, they have the ability to reconstruct themselves if an obstacle is placed along the propagation axis. Durnin et al. ${ }^{60}$ studied for the first time these kinds of beams, namely, the Bessel beams. However, so far, little attention has been paid to DF-SPPBs. The reason being, at least partially, that it has been shown that the Airy beam is the only possible diffraction-free solution to the 2D paraxial wave equation. ${ }^{61}$ Nevertheless, a possible generation of DF-SPPBs by means of axicon-shaped arrays of metal nanoparticles has been numerically predicted.$^{43}$ Inspired by this, we have proposed and demonstrated experimentally a simple structure for the generation of DF-SPPBs utilizing the SPP excitation by metal ridges illuminated at normal incidence. ${ }^{62}$ The structure was fabricated on a $50 \mathrm{~nm}$ thin gold film supported by a glass substrate in order to generate the DF-SPPBs targeting the excitation wavelength of $700 \mathrm{~nm}$. Such structure consists of two gold ridges $(300 \mathrm{~nm}$ wide, $70 \mathrm{~nm}$ thick, and $15 \mu \mathrm{m}$ long, each) joined at one of their ends forming an angle $\theta$. The ridges were fabricated using a common procedure of electron-beam lithography followed by liftoff. A $0.17 \mathrm{~mm}$ thin glass cover slip was used as a substrate to hold the sample. The LRM image of the generated DF-SPPBs closely resembles the zeroth-order Bessel function of the first kind [Fig. 10(a)]. The generated beams exhibited a negligible divergence $(\sim 8 \pi \mathrm{mrad})$ and a full width at half-maximum (FWHM) of $1.1 \mu \mathrm{m}(\sim 1.5 \lambda)$ at the point where the beam intensity has its maximum value [Fig. 10(b)]. Therefore, in general, we can claim that we have mimic the functionality of an optical axicon in order to generate limited-diffraction plasmonic beams with the zeroth-order Bessel intensity profiles within a limited region.

\section{Outlook}

In this paper, relevant experimental results concerning SPP propagation and control at subwavelength scale along with proper numerical

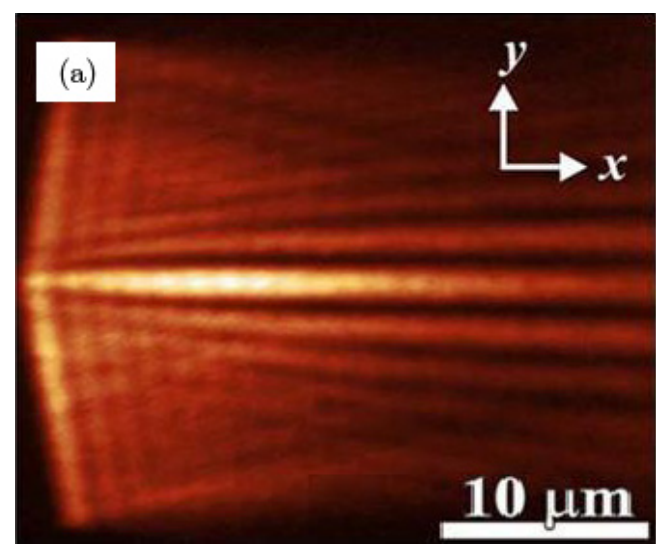

(a)

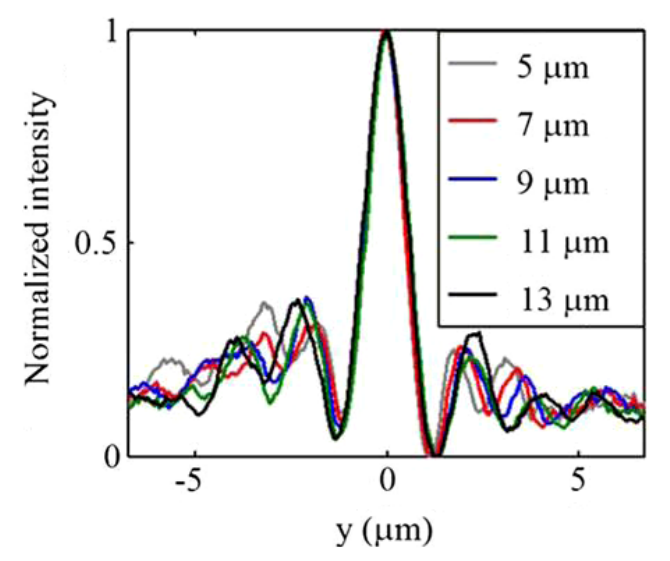

(b)

Fig. 10. LRM images of DF-SPPBs generated by an axicon like-nanostructure. (b) Averaged cross-sections along the $y$-coordinate at different distances from the apex (origin) of the axicon like-nanostructure. 
simulations were reviewed. An enormous advance, in this context, was achieved with the use of SNOM techniques. For example, features such as the influence of the SPP inelastic scattering were clearly analyzed and evaluated. The fabrication of SPP micro scatterers was pointed out, and their use in the construction of the basis of a 2D SPP optics was investigated. Furthermore, the possibility of simultaneous excitation, propagation and manipulation of SPP modes was presented. The results showed the feasibility to manipulate SPPs without using external excitation elements as for example an incoupling ridge. One should notice that analogue launching mechanisms are already adopted in plasmonic waveguides. As far as SPP guiding is concerned, it should be stressed that there are many basic and technological aspects that have to be yet explored. The physics behind such a phenomenon is not simple, in fact, although the optical energy would be well confined in a plasmonic waveguide; the propagation length will be reduced because of the Ohmic loss in metals. It was established that such usual trade-off can be improved by the use of DLSPPW. We show that dielectric loaded WRTRs structures can exhibit high extinction ratio, good mode confinement and relatively low losses in the near-infrared wavelength range. On the other hand, there is a class of diffraction-free mode solutions of the Helmholtz equation, which describes well-defined beams with narrow beam radii. The use of such beams might help to deliver a SP signal toward a desired point without the use of waveguides. In analogy with optical axicons, it was possible to generate limited-diffraction plasmonic beams with the zeroth-order Bessel intensity profiles within a limited region.

In general, the SPP propagation control at subwavelength scale is a very exciting field and many interesting results did not find their way into the above material, for example, the use of gain media as a mechanism to compensate ohmic loss. Additionally one can mention the progress in metamaterials, where it has been predicted that proper designed metasurfaces can fully manipulate SPPs in the near-field regime.

Finally, current experimental and theoretical investigations of classical plasmonic phenomena are indented to be the basis for miniaturization of photonics circuits with length scales much smaller than currently achievable, inter-chip and intra-chip applications in computer systems, and bio/sensor-system via the development of the labon-a chip concept.

\section{Acknowledgment}

The overall research was substantially benefited with discussions and very helpful reviews of S. I. Bozhevolnyi.

\section{References}

1. S. Maier, Plasmonics: Fundamentals and Applications (Springer, New York, 2007).

2. A. Maradudin, J. R. Sambles and W. Barnes (eds.), Modern Plasmonic (Elsevier, Amsterdam 2014).

3. L. Novotny and B. Hecht, Principles of NanoOptics, 2nd edn. (Cambridge University Press, New York, 2014).

4. W. Cai and V. Shalaev, Optical Metamaterials: Fundamentals and Applications (Springer, New York, 2014).

5. A. Dimitriev (ed.), Nanoplasmonic Sensors (Springer, New York, 2012).

6. H. Raether, Surface Plasmons, Springer Tracts in Modern Physics, Vol. 111 (Springer, Berlin, 1988).

7. E. H. Synge, Philos. Mag. 6, 356 (1928).

8. E. Ash and G. Nicholls, Nature 237, 510 (1972).

9. S. Kawata, M. Ohtsuhi and M. Irie (eds.), NanoOptics (Springer, Berlin, 2002).

10. V. Coello, Surf. Rev. Lett. 15, 867 (2008).

11. D. Courjon, Near-Field Microscopy and Near-Field Optics (World Scientific, Singapore, 2003).

12. S. I. Bozhevolnyi and V. Coello, Phys. Rev. B. 58, 10899 (1998).

13. I. I. Smolyaninov, D. L. Mazzoni, J. Mait and C. C. Davis, Phys. Rev. B 56, 1601 (1997).

14. I. I. Smolyaninov, D. L. Mazzoni and C. C. Davis, Phys. Rev. Lett. 77, 3877 (1996).

15. J. R. Krenn, R. Wolf, A. Leitner and F. R. Aussenegg, Opt. Commun. 137, 46 (1997).

16. S. C. Kitson, W. L. Barnes and J. R. Sambles, Phys. Rev. Lett. 77, 2670 (1997).

17. S. I. Bozhevolnyi and V. S. Volkov, Opt. Commun. 198, 241 (2001).

18. S. Randhawa, M. González, J. Renger, S. Enoch and R. Quidan, Opt. Exp. 14, 14496 (2010).

19. S. Bozhevolnyi, V. Volkov, K. Leosson and A. Boltasseva, J. Microsc. 209, 209 (2002).

20. G. Miano, G. R. Ubinacci and A. Tamburrino, Int. J. Appl. Electromagn. Mech. 35, 79 (2011).

21. R. Cortes and V. Coello, Nano: Brief Rep. Rev. 4, 201 (2009).

22. V. Coello, T. Søndergaard and S. I. Bozhevolnyi, Opt. Commun. 240, 345 (2004). 
23. H. Dittlbacher, J. R. Krenn, G. Shider, A. Leitner and F. R. Aussenegg, Appl. Phys. Lett. 81, 1762 (2002).

24. V. Coello, R. Villagomez, R. Cortes, R. Lopez and C. Martinez, Rev. Mex. Fis. 51, 426 (2005).

25. R. Cortés, V. Coello, R. Arriaga and N. Elizondo, Optik 125, 2400 (2014).

26. A. Drezet, A. Hohenau, A. Stepanov, H. Ditlbacher, B. Steinberger, N. Galler, F. R. Aussenegg, A. Leitner and J. R. Krenn, App. Phys. Lett. 89, 091117 (2006).

27. A. Drezet, D. Koller, A. Hohenau, A. Leitner, F. R. Aussenegg and J. R. Krenn, Opt. Lett. 32, 2414 (2007).

28. A. Drezet, A. Hohenau, D. Koller, A. Stepanov, H. Ditlbacher, B. Steinberger, F. R. Aussenegg, A. Leitner and J. R. Krenn. Mater. Sci. Eng. B 149, 220 (2008).

29. I. P. Radko, A. B. Evlyukhin, A. Boltasseva and S. I. Bozhevolnyi, Opt. Exp. 16, 3924 (2008).

30. A. Boltasseva, V. S. Volkov, R. B. Nielsen, E. Moreno, S. G. Rodrigo and S. I. Bozhevolnyi, Opt. Exp. 16, 5252 (2008).

31. D. Zhang, X. Yuan and A. Bouhelier, Appl. Opt. 49, 875 (2010).

32. V. Coello, R. Cortes, C. Garcia and N. Elizondo, Nano: Brief Rep. Rev. 8, 1350044 (2013).

33. I. Radko, S. Bozhevolnyi, G. Brucoli, L. MartínMoreno, F. J. García-Vidal and A. Boltasseva, Opt. Exp. 17, 7228 (2009).

34. V. Coello and S. I. Bozhevolnyi, Opt. Commun. 282, 3032 (2009).

35. C. Garcia, V. Coello, Z. Han, I. P. Radko and S. I. Bozhevolnyi, Appl. Phys. B 107, 401 (2012).

36. Z. Han, C. E. Garcia-Ortiz, I. P. Radko and S. I. Bozhevolnyi, Opt. Lett. 38, 875 (2013).

37. Z. Han and S. Bozhevolnyi, Rep. Prog. Phys. 76, 016402 (2013).

38. C. Garcia, V. Coello, Z. Han, I. P. Radko and S. I. Bozhevolnyi, Opt. Exp. 20, 7771 (2012).

39. J. Seidel, S. Grafström and L. Eng, Phys. Rev. Lett. 94, 177401 (2005).

40. M. Ambati, S. H. Nam, E. Ulin-Avila, D. A. Genov, G. Bartal and X. Zhang, Nano Lett. 8, 3998 (2008).

41. J. Grandidier, G. C. des Francs, S. Massenot, A. Bouhelier, L. Markey, J.-C. Weeber, C. Finot and A. Dereux, Nano Lett. 9, 2935 (2009).
42. P. Berini and I. De Leon, Nat. Photonics 6, 16 (2011).

43. I. P. Radko, V. S. Volkov, J. Beermann, A. B. Evlyukhin, T. Søndergaard, A. Boltasseva and S. I. Bozhevolnyi, Laser Photon. Rev. 3, 575 (2009).

44. A. Salandrino and D. N. Christodoulides, Opt. Lett. 35, 2082 (2010).

45. W. Liu, D. N. Neshev, I. V. Shadrivov, A. E. Miroshnichenko and Y. S. Kivshar, Opt. Lett. 36, 1164 (2011).

46. P. Zhang, S. Wang, Y. Liu, X. Yin, C. Lu, Z. Chen and X. Zhang, Opt. Lett. 36, 3191 (2011).

47. L. Li, T. Li, S. M. Wang, C. Zhang and S. N. Zhu, Phys. Rev. Lett. 107, 126804 (2011).

48. A. Minovich, A. E. Klein, N. Janunts, T. Pertsch, D. N. Neshev and Y. S. Kivshar, Phys. Rev. Lett. 107, 116802 (2011).

49. A. E. Klein, A. Minovich, M. Steinert, N. Janunts, A. Tünnermann, D. N. Neshev, Y. S. Kivshar and T. Pertsch, Opt. Lett. 37, 3402 (2012).

50. G. A. Siviloglou and D. N. Christodoulides, Opt. Lett. 32, 979 (2007).

51. H. Kurt and M. Turduev, J. Opt. Soc. Am. B 29, 1245 (2012).

52. V. Coello, S. I. Bozhevolnyi and F. Pudonin, Proc. SPIE. 3098, 536 (1997).

53. A. Kolomenski, A. Kolomenskii, J. Noel, S. Peng and H. Schuessler, Appl. Opt. 48, 30 (2009).

54. R. Carminati and J.-J. Greffet, Opt. Commun. 116, 316 (1995).

55. J. A. Marquez, R. Cortes, H. R. Siller, V. Coello and D. Escamilla, J. Appl. Res. Tech. 11, 886 (2013).

56. T. Søndergaard and S. I. Bozhevolnyi, Phys. Rev. B 67, 165405 (2003).

57. R. Cortes and V. Coello, Nano: Brief Rep. Rev. 4, 201 (2009).

58. T. Holmgaard and S. I. Bozhevolnyi, Phys. Rev. B 75, 245405 (2007).

59. A. Yariv, Electron. Lett. 36, 321 (2000).

60. J. Durnin, J. J. Miceli, Jr. and J. H. Eberly, Phys. Rev. Lett. 58, 1499 (1987).

61. G. A. Siviloglou and D. N. Christodoulides, Opt. Lett. 32, 979 (2007).

62. C. E. Garcia-Ortiz, V. Coello, Z. Han and S. I. Bozhevolnyi, Opt. Lett. 38, 905 (2013). 